\title{
Intrinsic decoherence effects on tripartite GHZ state generation using a trapped ion coupled to an optical cavity
}

\author{
S. Shelly Sharma* \\ Depto. de Fisica, Universidade Estadual de Londrina, Londrina 86040-370, PR Brazil \\ N. K. Sharmat \\ Depto. de Matematica, Universidade Estadual de Londrina, Londrina 86040-370 PR, Brazil
}

(Dated: November 30, 2018)

\begin{abstract}
We analyse the effects of intrinsic decoherence on the probability of generating a tripartite GHZ state using a cool trapped ion coupled to a single mode of the cavity field and interacting with a resonant laser field. Milburn equation is solved for this tripartite system to obtain the time evolution of density matrix as a function of cavity-ion coupling, laser-ion coupling, and the size of unitary time step relative to the time scale determined by system parameters. Starting with the system prepared initially in a separable state, density matrix is used to calculate the probability of tripartite GHZ state generation using coupling strengths reported in a recent experiment.
\end{abstract}

Interest in multipartite maximally entangled states is motivated by possible use in quantum communication [1], quantum dense coding [2] and quantum teleportation [3]. Advances in experimental techniques of trapping, cooling, preparation and manipulation of ionic quantum states [4, 5, 6], through interaction with external lasers, have made the generation of maximally entangled states a possibility. Coupling of trapped ion to quantized field inside an optical cavity has also been achieved 7]. With the ion trap placed inside a high finesse cavity, we have at hand a tripartite system with additional control mechanism offered by ion cavity coupling. This is the basic unit of a more complex multiple function system in which quantum states of several trapped ions placed coupled to an optical cavity may be manipulated in a controlled manner. In a letter [] , we proposed a scheme to generate three qubit maximally entangled GHZ state, using trapped ion interacting with a resonant external laser and sideband tuned single mode of a cavity field. Experimental implementation of such a proposal is constrained by the effects of quantum decoherence sources. Suppression of quantum coherence occurs due to random and unknown perturbations of system Hamiltonian. In a GHZ state generation experiment, the average effect of decoherence sources on probability of finding the tripartite system in GHZ state is of interest. In this paper, we investigate the effects of intrinsic decoherence on the composite system dynamics using Milburn model [9] $]$. Milburn model of intrinsic decoherence is based on the assumption that a closed quantum system evolves in a stochastic sequence of identical unitary transformations. The size of the unitary step along with the energy spectrum of the quantum system, determine the decoherence rate. The model applied by Milburn to free evolution of a closed quantum system, has since been used to study decoherence effects in JCM model with a single quantized field mode [10, 11], decoherence in multiphoton JCM model 12], decoherence of open systems [13], decoherence of a two-level particle coupled to photon field [14] and decoherence of quantum gate [15]. We solve the Milburn equation exactly for a cool trapped ion coupled to a single mode of the cavity field and interacting with a resonant laser field. The evolution dynamics of tripartite system is a function of laser-ion coupling, cavity ion coupling, and size of unitary step relative to the time scale determined by system parameters. The system is prepared initially in a separable state having ionic center of mass and cavity mode in respective number states, while the ion is in ground state. From the density matrix containing intrinsic decoherence effects, we calculate the probability of tripartite GHZ state generation using coupling strengths reported in recent experiments []].

Cold ions in a linear trap [4, 5, 6] offer a promising physical system for quantum information processing, as each ion allows two qubit state manipulation. With an ion trap placed inside a high finesse optical cavity, we obtain a three qubit system with additional control mechanism offered by quantized cavity field. The interaction picture Hamiltonian for this system is given in a detailed form in Eq. (4) of ref. [16]. On the time scale involving only a few cycles of unitary time evolution, the effective dimensions of three subsystems namely ionic internal state $(A)$, state of center of mass motion $(B)$, and cavity field state $(C)$ depend not only on the ion type but also on the relevant part of the ion-field interaction. Presently, we consider the resonant interaction of a two level cold trapped ion having transition frequency $\omega_{0}$, with an external laser of frequency $\omega_{L}=\omega_{0}$, while the cavity field is tuned to red sideband of ionic vibrational motion, $\omega_{0}-\omega_{c}=\nu$. Here $\nu$ is the frequency of one dimensional harmonic oscillator trapping potential. The Lamb-Dicke (LD) parameters relative to the laser field and the cavity field are denoted by $\eta_{L}$ and $\eta_{c}$ respectively. Working in the Lamb-Dicke regime, $\eta_{L} \ll 1$ and $\eta_{c} \ll 1$, the dynamics of the trapped two-level ion is

\footnotetext{
*shelly@uel.bı shelly@uel.br

${ }^{\dagger}$ nsharma@uel.br Your e-mail address
} 
governed by interaction Hamiltonian,

$$
\hat{H}_{I}=\hbar \Omega\left[\sigma_{+}+\sigma_{-}\right]+\hbar g \eta_{c}\left[\sigma_{+} \hat{b} \hat{a}+\sigma_{-} \hat{b}^{\dagger} \hat{a}^{\dagger}\right]
$$

where $\hat{a}^{\dagger}(\hat{a})$ and $\hat{b}^{\dagger}(\hat{b})$ are creation (annihilation) operators for vibrational phonon and cavity field photon respectively. The ion-phonon and ion-cavity coupling constants are $\Omega$ and $g$, whereas $\sigma_{k}(k=z,+,-)$ are the Pauli operators qualifying the internal state of the ion.

To obtain time evolution of the system, we work in the basis $|g, m, n\rangle,|e, m, n\rangle,|g, m-1, n-1\rangle$, and $|e, m-1, n-1\rangle$. Here $m, n=0,1, . ., \infty$ denote the state of ionic vibrational motion and quantized cavity field, respectively. The matrix representation of interaction operator $\hat{H}_{I}$ in the chosen basis, to the lowest order in $\eta_{L}$ and $\eta_{c}$, is

$$
H_{I}=\left[\begin{array}{cccc}
0 & \hbar \Omega & 0 & \hbar g \eta_{c} \sqrt{m n} \\
\hbar \Omega & 0 & 0 & 0 \\
0 & 0 & 0 & \hbar \Omega \\
\hbar g \eta_{c} \sqrt{m n} & 0 & \hbar \Omega & 0
\end{array}\right]
$$

The unitary transformation that diagonalizes $H_{I}$ is easily obtained and yields the eigenvectors, $\widehat{H}_{I} \Phi_{p}=E_{p} \Phi_{p}$, $(p=1,4)$. Milburn [9] postulates that on sufficiently short time steps, the quantum system evolves stochastically and the change in density operator in a time interval $(t, t+\tau)$ is given by

$$
\widehat{\rho}(t+\tau)=\gamma \exp \left(-\frac{i}{\hbar} \theta(\tau) \widehat{H}_{I}\right) \widehat{\rho}(t) \exp \left(\frac{i}{\hbar} \theta(\tau) \widehat{H}_{I}\right),
$$

$\theta(\tau)$ being some function of $\tau$. In standard quantum mechanics $\theta(\tau)=\tau$. In Milburn model $\lim _{\tau \rightarrow 0} \theta(\tau)=1 / \gamma$, a constant minimum time step with mean frequency $\gamma$. The evolution of density operator $\widehat{\rho}(t)$ in Milburn model , is governed by the equation

$$
\frac{d}{d t} \widehat{\rho}(t)=\gamma\left[\exp \left(-\frac{i}{\hbar \gamma} \widehat{H}_{I}\right) \widehat{\rho}(t) \exp \left(\frac{i}{\hbar \gamma} \widehat{H}_{I}\right)-\widehat{\rho}(t)\right]
$$

and the rate of change of density operator to first order in $\gamma^{-1}$ as obtained from Eq. (4) is

$$
\frac{d}{d t} \widehat{\rho}(t)=-\frac{i}{\hbar}\left[\widehat{H}_{I}, \widehat{\rho}(t)\right]-\frac{1}{2 \hbar^{2} \gamma}\left[\widehat{H}_{I},\left[\widehat{H}_{I}, \widehat{\rho}(t)\right]\right] .
$$

Transforming to the basis in which $\widehat{H}_{I}$ is diagonal and integrating Eq. (5), we get

$$
\langle\widehat{\rho}(t)\rangle=\sum_{p, q=1,4}\langle\widehat{\rho}(t)\rangle_{p q}\left|\Phi_{p}\right\rangle\left\langle\Phi_{q}\right|
$$

where

$$
\langle\widehat{\rho}(t)\rangle_{p q}=\langle\widehat{\rho}(0)\rangle_{p q} \exp \left(-\frac{i\left(E_{p}-E_{q}\right) t}{\hbar}-\frac{\left(E_{p}-E_{q}\right)^{2} t}{2 \hbar^{2} \gamma}\right)
$$

The presence of factors of the type $\left(E_{p}-E_{q} / \hbar\right)^{2}$ in the exponential indicates that energy spectrum of interaction operator $\widehat{H}_{I}$ plays an important role in determining the intrinsic decoherence rate. By solving Eq. (6), for the initial state $\widehat{\rho}(0)=|g, m-1, n-1\rangle\langle g, m-1, n-1|$, we get

$$
\begin{aligned}
\widehat{\rho}(t)= & \frac{(A+B)^{2}}{2}\left[\left|\Phi_{1}\right\rangle\left\langle\Phi_{1}|+| \Phi_{4}\right\rangle\left\langle\Phi_{4}\right|\right] \\
& -\frac{(A+B)^{2}}{2}\left[\exp \left(-\frac{2\left(\mu_{m n}-a_{m n}\right)^{2} t}{\gamma}-i 2\left(\mu_{m n}-a_{m n}\right) t\right)\left|\Phi_{1}\right\rangle\left\langle\Phi_{4}\right|\right. \\
& \left.+\exp \left(-\frac{2\left(\mu_{m n}-a_{m n}\right)^{2} t}{\gamma}+i 2\left(\mu_{m n}-a_{m n}\right) t\right)\left|\Phi_{4}\right\rangle\left\langle\Phi_{1}\right|\right]
\end{aligned}
$$


TABLE I: $P_{G H Z}(t)$ peak values at $T=(\pi / 4,3 \pi / 4)$, for $a_{11} / \gamma=0.001,0.005,0.01,0.1$.

\begin{tabular}{|c|r|r|r|}
\hline \hline \multicolumn{2}{|c|}{$\frac{1}{\gamma}$} & $P_{G H Z}$ & $P_{G H Z}$ \\
\hline$\left(=a_{11} / \gamma\right)$ & (in nano sec) & $T=\pi / 4$ & $T=3 \pi / 4$ \\
\hline \multicolumn{2}{|c|}{ No intrinsic decoherence } & 1.0 & 1.0 \\
\hline 0.001 & 0.43 & 0.99 & 0.94 \\
\hline 0.005 & 2.15 & 0.94 & 0.78 \\
\hline 0.01 & 4.32 & 0.89 & 0.65 \\
\hline 0.1 & 43.20 & 0.53 & 0.37 \\
\hline \hline
\end{tabular}

$$
\begin{aligned}
& +\frac{(A-B)^{2}}{2}\left[\exp \left(-\frac{2\left(\mu_{m n}+a_{m n}\right)^{2} t}{\gamma}+i 2\left(\mu_{m n}+a_{m n}\right) t\right)\left|\Phi_{2}\right\rangle\left\langle\Phi_{3}\right|\right. \\
& \left.+\exp \left(-\frac{2\left(\mu_{m n}+a_{m n}\right)^{2} t}{\gamma}-i 2\left(\mu_{m n}+a_{m n}\right) t\right)\left|\Phi_{3}\right\rangle\left\langle\Phi_{2}\right|\right] \\
& \frac{(A-B)^{2}}{2}\left(\left|\Phi_{2}\right\rangle\left\langle\Phi_{2}|+| \Phi_{3}\right\rangle\left\langle\Phi_{3}\right|\right) \\
& +\frac{\left(A^{2}-B^{2}\right)}{2}\left[\exp \left(-\frac{2 \mu_{m n}^{2} t}{\gamma}-i 2 \mu_{m n} t\right)\left(\left|\Phi_{1}\right\rangle\left\langle\Phi_{2}|-| \Phi_{3}\right\rangle\left\langle\Phi_{4}\right|\right)\right. \\
& +\exp \left(-\frac{2 \mu_{m n}^{2} t}{\gamma}+i 2 \mu_{m n} t\right)\left(\left|\Phi_{2}\right\rangle\left\langle\Phi_{1}|-| \Phi_{4}\right\rangle\left\langle\Phi_{3}\right|\right) \\
& +\exp \left(-\frac{2 a_{m n}^{2} t}{\gamma}+i 2 a_{m n} t\right)\left(\left|\Phi_{1}\right\rangle\left\langle\Phi_{3}|-| \Phi_{2}\right\rangle\left\langle\Phi_{4}\right|\right) \\
& \left.+\exp \left(-\frac{2 a_{m n}^{2} t}{\gamma}-i 2 a_{m n} t\right)\left(\left|\Phi_{3}\right\rangle\left\langle\Phi_{1}|-| \Phi_{4}\right\rangle\left\langle\Phi_{2}\right|\right)\right]
\end{aligned}
$$

where $a_{m n}=\frac{1}{2} g \eta_{c} \sqrt{m n}, \mu_{m n}=\sqrt{a_{m n}^{2}+\Omega^{2}}, A^{2}=\left(\mu_{m n}+\Omega\right) / 4 \mu_{m n}$, and $B^{2}=\left(\mu_{m n}-\Omega\right) / 4 \mu_{m n}$.

Next we consider a special case with the ion in its ground state occupying the lowest energy trap state, while the cavity is prepared in vacuum state. The density operator for the initial state is $\widehat{\rho}(0)=|g, 0,0\rangle\langle g, 0,0|,(m=1, n=1)$. Without taking into consideration the decoherence effects, the interaction time $t_{p}$ needed to generate the maximally entangled tripartite GHZ state depends on the the ratio $\alpha=\left(\mu_{11} / a_{11}\right)$, involving parameters characterizing the ionlaser and ion-cavity interaction strengths. For interaction time $t_{p}$ such that $a_{11} t_{p}=\frac{\pi}{4}, \mu_{11} t_{p}=p \pi, p=1,2, \ldots,(\alpha=4)$ the system is found to be in maximally entangled tripartite two mode GHZ state []ㅁ]

$$
\Psi_{G H Z}=\frac{(-1)^{p}}{\sqrt{2}}(|g, 0,0\rangle-i|e, 1,1\rangle) .
$$

Using Eq. (7), we can easily write down the density operator $\widehat{\rho}(t)$ for $\widehat{\rho}(0)=|g, 0,0\rangle\langle g, 0,0|$. Next we evaluate the probability $P_{G H Z}(t)=\operatorname{tr}\left(\widehat{\rho}(t) \widehat{\rho}_{G H Z}\right)$ of finding the system in the state $\widehat{\rho}_{G H Z}=\left|\Psi_{G H Z}\right\rangle\left\langle\Psi_{G H Z}\right|$ and obtain

$$
\begin{aligned}
P_{G H Z}(t)= & \frac{1}{2} \cdot+\frac{\Omega^{2}}{2 \mu_{11}^{2}}\left(\exp \left(\frac{-2 \mu_{11}^{2} t}{\gamma}\right) \cos \left(2 \mu_{11} t\right)\right) \\
& +\frac{\Omega^{2}}{2 \mu_{11}^{2}}\left(\exp \left(\frac{-2 a_{11}^{2} t}{\gamma}\right) \sin \left(2 a_{11} t\right)-1\right) \\
& +\frac{1}{2}\left(\frac{\mu_{11}-a_{11}}{2 \mu_{11}}\right)^{2} \exp \left(\frac{-2\left(\mu_{11}+a_{11}\right) t}{\gamma}\right) \sin \left[2\left(\mu_{11}+a_{11}\right) t\right] \\
& -\frac{1}{2}\left(\frac{\mu_{11}+a_{11}}{2 \mu_{11}}\right)^{2} \exp \left(\frac{-2\left(\mu_{11}-a_{11}\right) t}{\gamma}\right) \sin \left[2\left(\mu_{11}-a_{11}\right) t\right] .
\end{aligned}
$$

In the limit $a_{11} / \gamma \rightarrow 0$ corresponding to continuous time evolution, Eq. (7) and Eq. (9) reduce to decoherence free time evolution of density operator and $P_{G H Z}$ respectively. Fig. 1 displays $P_{G H Z}(t)$ as a function of scaled time 


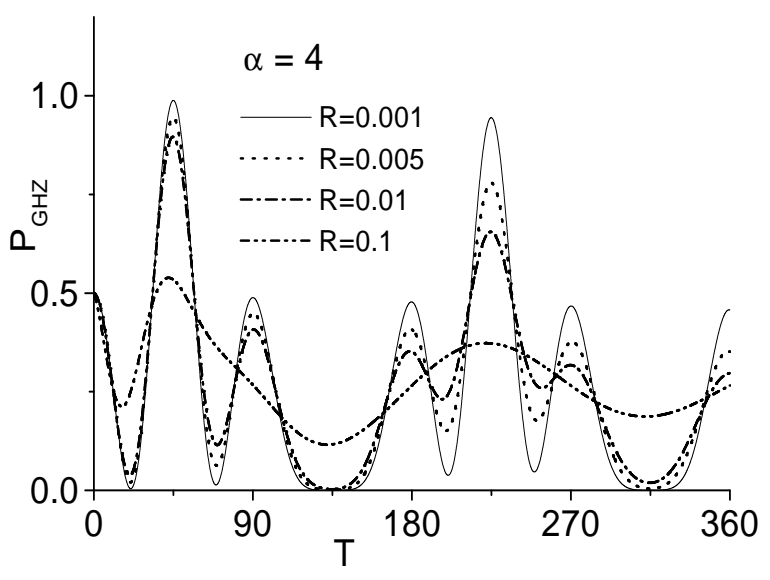

FIG. 1: $P_{G H Z}(t)$ as a function of scaled time variable $T\left(=a_{11} t\right)$ in degrees, for the choice $\mu_{11} \backslash a_{11}=4$, and intrinsic decoherence parameter $R=a_{11} / \gamma=0.001,0.005,0.01,0.1$.

variable $T\left(=a_{11} t\right)$, for the choice $\mu_{11} \backslash a_{11}=4$, and intrinsic decoherence parameter $R=a_{11} / \gamma=0.001,0.005,0.01,0.1$. Evidently the evolution dynamics of $P_{G H Z}(t)$ is sensitive to changes in intrinsic decoherence parameter $R$. With increase in the value of $R$ the peaks in $P_{G H Z}(t)$ plots are seen to get lower. Using the value $\Omega=8.95 \mathrm{MHz}$ [] and recalling that $\mu_{m n}=\sqrt{a_{m n}^{2}+\Omega^{2}}$, we calculate the mean frequency $\gamma$ of minimum time step. Table 1 . lists the $P_{G H Z}(t)$ peak values at $T=\pi / 4,3 \pi / 4$, for the choices $a_{11} / \gamma=0.001,0.005,0.01,0.1$. As expected, the presence of decay factors in Eq. (9) causes the $P_{G H Z}(t)$ peak values to decrease with increase in $1 / \gamma$. For $a_{11} / \gamma=0.1$, decoherence effects dominate the scene resulting in a low $P_{G H Z}(t)$ peak value of 0.53 . We may recall here that for the coupling value determined by $\Omega=8.95 \mathrm{MHz}$, the angle $T=\pi / 4$ corresponds to an interaction time of $t_{1} \sim 0.34 \mu \mathrm{s}$. The probability of finding the system in GHZ state can be measured by cavity-photon measurement combined with atomic population inversion measurement.

\section{CONCLUSIONS}

We have analysed the evolution dynamics of a single two-level trapped ion coupled to an optical cavity interacting with a laser field and a single cavity mode. Assuming that the quantum system evolves in a stochastic sequence of identical unitary transformations [9], the probability of generating a maximally entangled tripartite GHZ state has been calculated. We have used intraction parameters reported in a recent experiment 7]. As expected, the presence of decay factors in time evolution equation, causes the $P_{G H Z}(t)$ peak values to decrease with increase in the average size of the minimum time step. The evolution dynamics of $P_{G H Z}(t)$ is also sensitive to changes in intrinsic decoherence parameter $R$. Besides that the energy spectrum of system hamiltonian determines the decoherence rate for a given initial state preparation. For the case at hand, that is ion initially in its ground state occupying the lowest energy trap level, while the cavity is prepared in vacuum state, the ratio $R=\left(\frac{1}{2} g \eta_{c}\right) / \gamma$ is crucial in determining the rate at which GHZ state generation probability $P_{G H Z}(t)$, decays with time. Even for a large value of $a_{11} / \gamma=0.01$, we obtain GHZ state generation probability of 0.89 . Decoherence effects due to cavity decay, heating and other random sources of decoherence on $P_{G H Z}$ have not been considered.

[1] A. K. Ekert, Phys. Rev. Lett. 67, 661 (1991).

[2] C. H. Bennett and S. J. Wiesner, Phys. Rev. Lett. 69, 2881 (1992).

[3] C. H. Bennett, G. Brassard, C. Crepeau, R. Jozsa, A. Peres, and W. K. Wootters, Phys. Rev. Lett. 70, 1895 (1993).

[4] C. Monroe, D.M. Meekhof, B.E. King, W.M Itano and D.J. Wineland, Phys. Rev. Lett. 75(1995)

[5] D.J. Wineland, C. Monroe, W.M Itano, D. Leibfried, B.E. King, and D.M. Meekhof, NIST J. Res. 103, 259 (1998)

[6] C. Roos, Th. Zeiger, H. Rohde, H. C. Nagerl, J. Eschner, D. Liebfried, F. Schmidt-Kaler, R. Blatt, Phys.Rev. Lett. 83, 4713 (1999).

[7] A. B. Mundt, A. Kreuter, C. Russo, C. Becher, D. Leibfried, J. Eschner, F. Schmidt-Kaler, R. Blatt, in Appl. Phys. B 76 (2003) 117.

[8] S. Shelly Sharma, Phys. Letts. A 311 (2003) 111.

[9] G. J. Milburn, Phys. Rev. A 44 (1991) 5401. 
[10] H. Moya-Cessa, V. Buzek, M. S. Kim, P. L. Knight, Phys. Rev. A 48 (1993) 3900.

[11] M. B. Plenio, P. L. Knight, Proc. R. Soc. A 453 (1997) 2017.

[12] L. M. Kuang, X. Chen, and M. L. Ge, Phys. Rev. A 52 (1995) 1857.

[13] V. Buzek and M. Konopka, Phys. Rev. A 58 (1998) 1735.

[14] Nour A. Zidan, Mahmoud Abdel-Aty and Abdel-shafy F. Obada, Chaos Solitons and Fractals, 13 (2002) 1421.

[15] K. Kimm and Hwang-h. Kwon, Phys. Rev. A 65, 022311(2002)

[16] S. Shelly Sharma, and A. Vidiella-Barranco, Phys. Lett. A 309 (2003) 345. 